\title{
Intra-operative Coronary Subclavian Steal Syndrome
}

\author{
Sam Mann ${ }^{1}$, Katharine Tweed ${ }^{2}$, Marius Berman ${ }^{2}$, and Jason Ali ${ }^{2}$ \\ ${ }^{1}$ Papworth Hospital NHS Foundation Trust \\ ${ }^{2}$ Royal Papworth Hospital
}

April 28, 2020

\begin{abstract}
Coronary-subclavian steal syndrome (CSSS) describes the condition whereby haemodynamically significant proximal subclavian artery stenosis results in flow limitation or reversal within the left internal mammary artery (LIMA) graft, classically precipitated by upper limb exertion. We report an unusual presentation, where a patient developed pronounced ST-depression and haemodynamic instability immediately following weaning from bypass. The patient was subsequently found to have occlusion of their left subclavian artery. Despite its incidence, there are currently no recommendations for screening for subclavian artery stenosis. We propose a preoperative screening algorithm that may allow for intervention prior to CABG, enabling risk-free use of the LIMA.
\end{abstract}

\section{Keywords}

Coronary subclavian steal syndrome, left internal mammary, CABG

\section{Introduction}

The left internal mammary artery (LIMA) is the preferred conduit for coronary artery bypass surgery $(\mathrm{CABG})$. Originating from the left subclavian artery, the LIMA is threatened by proximal subclavian artery stenosis (SAS). Coronary subclavian steal syndrome (CSSS) describes the condition whereby haemodynamically significant proximal SAS results in flow limitation or reversal within the LIMA graft, classically presenting as myocardial ischemia precipitated by upper limb exertion a number of months following CABG and occurs in as many as $6.8 \%$ of patients[1].

\section{Case report}

A 48-year old female was referred for surgical revascularisation. She had been admitted with worsening angina and increasing exertional dyspnoea. She had suffered a non-ST elevation myocardial infarction 1year prior to referral and had her left main stem stented. Her past medical history included: obesity and a right hip replacement. She was an ex-smoker with a 15-pack-year history and had a strong family history of ischaemic heart disease.

Her angiogram confirmed significant in-stent restenosis of the left main stem, with $50 \%$ stenosis and an instant flow reserve (iFR) 0.82, and a severe ostial left circumflex lesion. Echocardiogram confirmed good left ventricular function and no significant valvular pathology.

The patient underwent CABG x 2. Prior to establishing bypass, the pedicled LIMA flow was assessed as reasonable, at high pressures, and was subsequently anastomosed to the left anterior descending (LAD) artery. There was myocardial contraction observed upon release of LIMA flow suggesting no technical issue with the anastomosis. The heart was subsequently weaned from cardiopulmonary bypass with no inotropic support. Shortly after, pronounced anterolateral ST-depression was observed followed by haemodynamic instability prompting going back onto bypass. 
The LIMA-LAD anastomosis was taken down in view of the pattern of ischaemia. Good flow was observed from the LIMA and there was no evidence of a technical problem, however the decision was made to perform a vein graft to the LAD. The LIMA was not used as a free graft as there was concern that there may have been an injury to the vessel as the cause of the problem. The remainder of the procedure proceeded uneventfully, and she was transferred to the ITU with no inotropic support. To further investigate, a CT-aortogram was performed which confirmed complete occlusion of the proximal left subclavian artery at its origin (Figure 1 ), suggesting the intraoperative picture was that of coronary-subclavian steal syndrome. She was treated with antibiotics for a chest infection, but otherwise made an uneventful recovery and was discharged on the 7th postoperative day.

\section{Comment}

CSSS is estimated to complicate between $0.2-6.8 \%$ of CABG operations[1] and is due to subclavian artery stenosis (SAS) or occlusion which has been reported to be present in up to $5.3 \%$ of patients undergoing CABG, and in $11.8 \%$ of those with a history of peripheral vascular disease (PVD) [2]. It typically presents after surgery with symptoms ranging from stable angina to acute coronary syndrome and even sudden cardiac death that may occur many months postoperatively usually following left arm exertion[3].

Here we report an unusual presentation of CSSS, presenting intraoperatively with haemodynamic instability after weaning from CPB, presumably representing myocardial ischaemia due to inadequate LIMA flow. We hypothesise that this was due to the change in coronary vascular resistance occurring. With an unloaded heart on $\mathrm{CPB}$, there is a low resistance vascular bed offering minimal resistance to LIMA flow throughout the cardiac cycle, but upon weaning from CPB there is increased coronary vascular resistance[4] and only diastolic perfusion which was presumably compromised due to the collateral origin of the LIMA. This would also explain why upon taking down the artery there was apparently good flow from the LIMA. Given the apparent unreliability of the LIMA graft, the decision was made to use a vein graft instead.

In view of the relatively high incidence of SAS in the CABG population, it is surprising that there are no recommendations on screening for this problem in guidelines on coronary revascularisation, especially in the era of increasing use of bilateral internal mammary arteries. There are two relatively inexpensive options for screening: i) measurement of bilateral brachial blood pressure - where a difference in systolic pressure of 15 or $20 \mathrm{mmHg}$ has been suggested to be diagnostic of haemodynamically significant SAS; and ii) ultrasound doppler - which is often performed to exclude carotid artery disease, where systolic vertebral artery flow reversal is suggestive of haemodynamically significant SAS. In patients with suspicion of SAS, diagnostic options include computed tomography or magnetic resonance angiography, or digital subtraction angiography, and following confirmation of diagnosis there is opportunity to intervene and treat the problem, for example with stenting, to minimise the risk of CSSS postoperatively[5].

In our patient, subsequent measurement of bilateral brachial artery pressures identified a $30 \mathrm{mmHg}$ difference in systolic pressure and would have alerted to the presence of SAS had this been performed preoperatively. As such we believe there may be some value in screening patients pre-operatively and are now introducing bilateral brachial blood pressure measurement as a routine screening for SAS in patients undergoing CABG and propose an algorithm (Figure 2).

\section{Conclusion}

This case demonstrates an unusual presentation of CSSS intraoperatively. We would recommend considering SAS particularly in patients with significant atherosclerotic risk factors and peripheral vascular disease. We propose a preoperative screening algorithm that may allow for intervention prior to CABG, enabling risk-free use of the LIMA and improving postoperative outcomes.

\section{References}

[1] Iglesias JF, Degrauwe S, Monney P, Glauser F, Qanadli SD, Eeckhout E, et al. Coronary Subclavian Steal Syndrome and Acute Anterior Myocardial Infarction: A New Treatment Dilemma in the Era of Primary Percutaneous Coronary Intervention. Circulation 2015;132:70-1. doi:10.1161/CIRCULATIONAHA.115.015226. 
[2] English JA, Carell ES, Guidera SA, Tripp HF. Angiographic prevalence and clinical predictors of left subclavian stenosis in patients undergoing diagnostic cardiac catheterization. Catheterization and Cardiovascular Interventions : Official Journal of the Society for Cardiac Angiography \& Interventions 2001;54:8-11. doi:10.1002/ccd.1230.

[3] De Roeck F, Tijskens M, Segers VFM. Coronary-subclavian steal syndrome, an easily overlooked entity in interventional cardiology. Catheterization and Cardiovascular Interventions : Official Journal of the Society for Cardiac Angiography \& Interventions 2019;70:I213. doi:10.1002/ccd.28362.

[4] Belboul A, Rådberg G, Roberts D, Dernevik L. Intraoperative assessement of coronary flow and coronary vascular resistance during coronary bypass surgery. Scand Cardiovasc J 1999;33:23-8.

[5] Cua B, Mamdani N, Halpin D, Jhamnani S, Jayasuriya S, Mena-Hurtado C. Review of coronary subclavian steal syndrome. J Cardiol 2017;70:432-7. doi:10.1016/j.jjcc.2017.02.012.

\section{Figure Legends}

Figure 1. Computed tomography images a) contrast enhanced oblique view reformat and b) volume rendered 3D-reconstruction of the arterial tree demonstrating proximal occlusion of the left subclavian artery with thrombus.

Figure 2. Proposed algorithm for the screening of patients undergoing coronary artery bypass grafting for subclavian artery stenosis. CABG - coronary artery bypass grafting, CTA - computed tomography angiography, MRA - magnetic resonance angiography

\section{Hosted file}

Subclavian stenosis figure.pptx available at https://authorea.com/users/312825/articles/443394intra-operative-coronary-subclavian-steal-syndrome

\section{Hosted file}

Subclavian stenosis figure 2.pptx available at https://authorea.com/users/312825/articles/443394intra-operative-coronary-subclavian-steal-syndrome 